\title{
Analytical Modelling of Dielectric Modulated Negative Capacitance MoS2-FET for Biosensor Application
}

\author{
D. K. Panda ${ }^{1}$, R. Singh ${ }^{2}$, T. R. Lenka ${ }^{2}$, V. Goyal ${ }^{3}$, N. El. I. Boukortt ${ }^{4}$ and H. P. T. Nguyen ${ }^{5}$
}

\begin{abstract}
In this paper, a dielectric modulated negative capacitance (NC)-MoS $\mathrm{S}_{2}$ field effect transistor (FET)-based biosensor is proposed for label-free detection of biomolecules such as enzymes, proteins, DNA, etc. Various reports present experimental demonstration and modelling of $\mathrm{NC}-\mathrm{MoS}_{2} \mathrm{FET}$, but it is never utilized as a dielectric modulated biosensor. Therefore, in this work, the modelling, characterization and sensitivity analysis of dielectric modulated $\mathrm{NC}-\mathrm{MoS} \mathrm{S}_{2}$ FET is focussed. For immobilization of biomolecules, a nanocavity is formed below the gate by etching some portion of the gate oxide material. The immobilization of biomolecules in the cavity leads to a variation of different electrostatic properties such as surface potential, threshold voltage, drain current, and subthreshold-swing (SS) which can be utilized as sensing parameters. An analytical model for the proposed biosensor is also developed in the subthreshold region by considering the properties of two-dimensional (2D) ferroelectric materials and benchmarked with TCAD device simulations. The effect of change of gate length and doping concentration on different electrical properties is also analysed to estimate the optimum value of channel doping. The results prove that the proposed device can be used for next-generation low power label-free biosensor which shows enhanced sensitivity as compared to traditional FET-based biosensors.
\end{abstract}
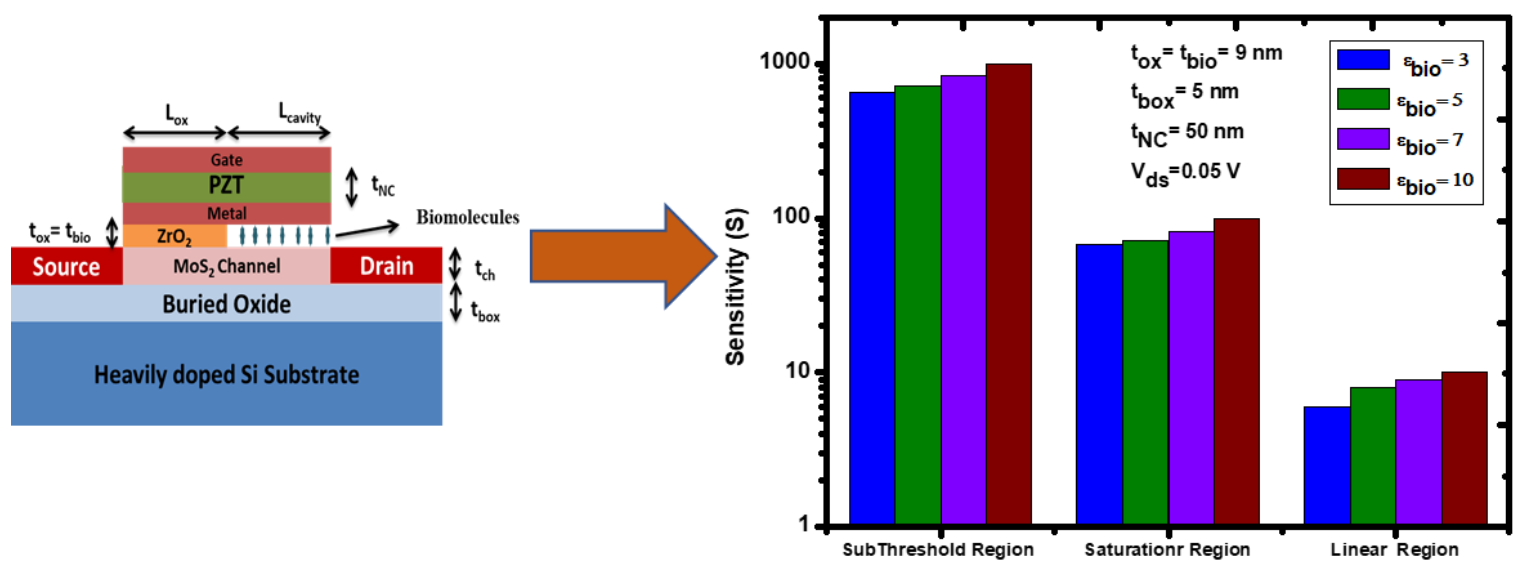

Keywords: Biosensor, $\mathrm{MoS}_{2}$, Negative Capacitance, Sensitivity, TCAD.

\section{INTRODUCTION}

B iosensors are applicable for medical and health care applications as well as for forensic industries, national security and monitoring of environment [1-5]. Among all types of biosensors, FET-based biosensor gains a lot of attention among researchers due to their highly desirable properties such as low power consumption, rapid detection of biomolecule without labelling, low-cost mass production, portability and easier for on chip integration [1-3]. One of the biggest challenges faced among the researchers in conventional FET based biosensors is their lower sensitivity [4]. In order to enhance the sensitivity of biosensors, different structural modification of conventional FET has been done and reported in literature [5-6]. Out of all these nanowire biosensors have shown great potential to become a practical detection platform in biological and medical applications.

${ }^{1}$ D. K. Panda is with the Microelectronics and VLSI Design Group, School of Electronics, VIT-AP University, Amaravati, AP, 522237 India. (d.panda.2016@ieee.org )

${ }^{2}$ R. Singh and T. R. Lenka are with the Microelectronics and VLSI Design Group, Department of Electronics \& Communication Engineering, National Institute of Technology Silchar, Assam, 788010, India. (rajan_rs@ece.nits.ac.in; trlenka@ieee.org )
${ }^{3} \mathrm{~V}$. Goyal is with Department of Electronics and Communication Engineering, GLA University, Mathura, UP, 281406, India. (vishal.goyal@gla.ac.in)

${ }^{4}$ Nour El I. Boukortt is with Electronics and Communication Engineering Department, Kuwait College of Science and Technology, Doha, Road N. 7, Kuwait (n.boukortt@kcst.edu.kw).

${ }^{5}$ H. P. T. Nguyen is with the Department of Electrical and Computer Engineering, New Jersey Institute of Technology, Newark, New Jersey, 07102, USA. (hieu.p.nguyen@ njit.edu )

Nanowire biosensors have various advantages, such as real-time transduction to electrical signal with high sensitivity and suitability of label-free detection. Although its sensitivity is very high as compared to other methods, its analytical signal intensity is very low which can be contaminated by high background noise. Hence, new channel materials are being continuously explored which can provide higher sensitivity and lower noise for next generation biosensors [6].

Apart from sensitivity, another most important parameter for evaluating the performance of the biosensors is the response time [7]. Response time is defined as the time required to obtain a desired sensitivity (time required to capture a certain surface density of biomolecules to achieve a desired change in electrical signal). Response time of biosensor can be reduced significantly by reducing SS of the 
device [7]. If the SS is lowered to obtain the same change in current and hence the same sensitivity, then the required change in surface potential is also lowered resulting lowering of the response time.

In order to cope with the demand of modern era of biosensors with the performance improvement, MOSFET scaling, response time and signal to noise ratio (SNR) are some of the important parameters to be considered $[5,7]$. The scale down of transistor and high density on-chip integration produces a lot of short channel effects (SCEs) which in turn dissipates a large amount of power in the device [5]. In order to design low power biosensor, the major challenges faced by the researchers are how to get rid of the SCEs. One of the possible techniques to overcome the SCEs in Si MOSFET is to incorporate different novel materials in the channel of FET. Out of different channel materials, two-dimensional (2D) transition metal chalcogenides $\left(\mathrm{MoS}_{2}, \mathrm{WS}_{2}\right.$, and $\mathrm{MoTe}_{2}$ ) have gained a lot of attention among world-wide researchers [8-11]. The 2D materials are helpful in better electrostatic control in the channel and aggressive scaling. Furthermore, by modifying the properties of 2D materials through defect engineering, 2D materials can be applied to selectively respond to particular analytes with enhanced sensitivity. These properties of $2 \mathrm{D}$ materials make nanomaterials suitable for next-generation biosensors for healthcare applications [12]. In particular $\mathrm{MoS}_{2}$ has great research interest due to its high $\mathrm{I}_{\text {on }} / \mathrm{I}_{\text {off }}$ ratio, high carrier mobility and larger intrinsic bandgap and lower SNR [1214]. With the advanced fabrication technologies highperformance 2D material based MOSFETs were fabricated with fully CMOS compatible processes [13].

In order to further reduce the power dissipation and SS of Nanoelectronics devices, ferroelectric materials can be explored which exhibit negative capacitance [15-18]. One promising alternative channel material is the $2 \mathrm{D}$ transition metal dichalcogenide (TMD, such as $\mathrm{MoS}_{2}$ ), which offers sub-nm thickness and a more stable channel capacitance that, when coupled with the NC-effect, could produce steep switching over a broad range of current [18]. One of the most important parameters of any switching device is how rapidly it changes its states from off-state to on-state. The SS parameter of the device can be used for measuring the above operation which in turn evaluates the power consumption. The SS of the FET can be formulated as $S S=$ $\partial V_{g s} / \partial \log _{10}\left(I_{d s}\right)$. When a ferroelectric material is incorporated, surface potential is developed within the ferroelectric layer and gate oxide. Subsequently, the above formula can be reformulated as $S S=\partial V_{g s} / \partial \psi_{s} \times$ $\partial \psi_{s} / \partial \log _{10}\left(I_{d s}\right)$. At room temperature, the second term of the above expression cannot be less than $60 \mathrm{mV} /$ decade due to the thermal Boltzmann limitation. But by using ferroelectric materials the SS can be further reduced below $60 \mathrm{mV} / \mathrm{dec}$ and hence power dissipation. In order to obtain lower SS different ferroelectric materials such as Hafnium Zirconium Oxide $\left(\mathrm{HfZrO}_{2}\right)$, Lead Zirconate Titanate (PZT), Al-doped $\mathrm{HfO}_{2}$ are being used by researchers. $\mathrm{MoS}_{2}$ MOSFET also shows NC effect as reported in different literatures [19-22]. However, to further improve the device performance, new technology and new device concepts need to be developed. Therefore, in this work a NC-MoS 2 FET is designed with improved on-off current ratio and utilized for the detection of different biomolecules.

In the literature, different types of $\mathrm{MoS}_{2}$ FET-based biosensors are reported [23-24]. They show excellent biodetection capability in subthreshold mode operation due to the 2D properties of $\mathrm{MoS}_{2}$ [23-24]. But the improved performance as reported in these biosensors are limited to charged biomolecule detection submerged in solution which suffers from electrostatic screening. In order to avoid these problems a new biomolecule detection technique has been reported in various literatures by using the change in gate dielectric permittivity instead of charge [25]. This type of biosensor can be implemented as dielectric modulated FET where biomolecules are placed inside the cavity formed in the oxide region [26-27]. Simulation and modelling of dielectric modulated FETs are reported in various literatures which are mostly used for sensing of biomolecules in dry environment [28-29]. In this manuscript a dielectric modulated negative capacitance $\mathrm{MoS}_{2}$ FET based biosensor is reported which can detect various biomolecules such as enzymes, proteins, DNAs etc.

Analytical and compact models act as a bridge between devices and circuits. The models are also helpful to understand the better insight of device operation and to evaluate the performance of biosensors. A lot of analytical and compact models of $\mathrm{MoS}_{2}$ FET and NC-MoS 2 FET are available in different literature [28-29], but as per best of author's knowledge till now no analytical or compact model for NC FET-based biosensors are available in literature. So in this manuscript an analytical model for NC-MoS 2 FET based biosensor is presented in subthreshold region by considering the impact of drain coupling through numerical simulations. A systematic investigation is performed of different sensitivity parameters of the biosensor such as change in threshold voltage, drain current, $\mathrm{I}_{\mathrm{on}} / \mathrm{I}_{\text {off }}$ ratio and SS through analytical model development.

The device structure of NC-MoS 2 FET along with different models used for device simulation are presented in Section-II followed by the development of an analytical model for the proposed biosensor in Section-III. The Results and Discussion is presented in Section-IV. Finally, the Conclusion is drawn in Section-V.

\section{Device StRucture And Simulation Model}

Fig.1 (a) shows device structure of dielectric modulated NC$\mathrm{MoS}_{2}$ MOSFET based biosensor. The thickness of $\mathrm{MoS}_{2}$ channel is considered as $2 \mathrm{~nm}$ [30]. In order to develop NC, a Lead Zirconate Titanate (PZT) layer is considered below the gate. Zirconium oxide $\left(\mathrm{ZrO}_{2}\right)$ of thickness $9 \mathrm{~nm}$ is utilized as the high- $K$ gate oxide material for reducing the gate leakage current. The channel doping concentration is varied to obtain the optimum doping concentration. Fig.1 (b) shows the corresponding equivalent capacitance circuit network of the device which is utilized for developing the analytical model. Since our model is not used for transient analysis, we do not consider the effect of resistance in our model. The channel length is considered as $40 \mathrm{~nm}$. The source and drain contact depths are considered as $9 \mathrm{~nm}$. The different device 
parameters used in the structure is presented in Table I. The device structure is proposed according to the rules of the fabricated device in [30].

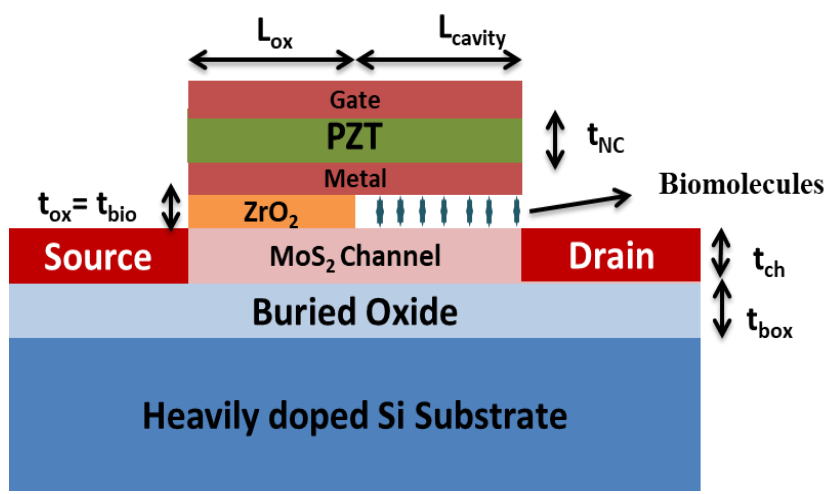

Fig. 1. (a) 2D Crosssectional view of NC-MoS 2 FET biosensor

In order to sense the different biomolecules, a portion of oxide layer is etched to form a cavity where appropriate bioreceptors are functionalized to provide binding of targeted biomolecules. When the targeted biomolecules are not present in the cavity region the holes are filled with air and hence the permittivity of the cavity region differs from the oxide layer and threshold voltage differs from its initial value. When the targeted biomolecules such as proteins, enzymes, DNAs etc with different relative dielectric constants $\left(\epsilon_{\text {bio }}\right)$ are put inside the cavity the effective capacitance of the device also varies. Consequently, different electrical characteristics such as surface potential, threshold voltage and drain current are changed as per the targeted biomolecules. For sensitivity analysis of the biosensor different dielectric constants of the biomolecules are considered such as $\epsilon_{\text {bio }}=3,5,7,10$ in the simulation. For simulating the device electric field dependent FLDMOB mobility model [31], concentration dependent KLASRH generation-recombination model [31] are used.

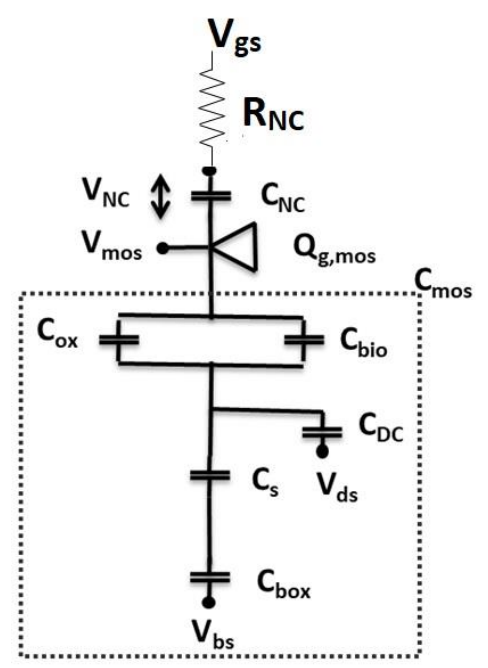

Fig. 1. (b) Equivalent capacitance network of the device
Table I Device Parameters Used For Simulation

\begin{tabular}{ll}
\hline Device Parameters & Values \\
\hline Channel Thickness [30] & $2 \mathrm{~nm}$ \\
Channel Length & $40 \mathrm{~nm}$ \\
Oxide thickness $\left(\mathrm{t}_{\mathrm{ox}}\right)=$ Biomolecule thickness $\left(\mathrm{t}_{\mathrm{bio}}\right)$ & $9 \mathrm{~nm}$ \\
Top oxide Length $\left(\mathrm{L}_{\mathrm{ox}}\right)$ & $20 \mathrm{~nm}$ \\
Nano Cavity length $\left(\mathrm{L}_{\mathrm{cavity}}\right)$ & $20 \mathrm{~nm}$ \\
Ferroelectric Thickness $\left(\mathrm{t}_{\mathrm{NC}}\right)$ & $50 \mathrm{~nm}$ \\
Buried oxide thickness (tbox $)$ & $5 \mathrm{~nm}$ \\
gate electrode work function & $4.5 \mathrm{eV}$ \\
$\mathrm{MoS}_{2}$ Bandgap energy & $1.8 \mathrm{eV}$ \\
$\mathrm{MoS}_{2}$ Dielectric constant & 7 \\
$\mathrm{MoS}_{2}$ Electron effective mass & 0.52 \\
$\mathrm{MoS}_{2}$ Hole effective mass & 0.64 \\
$\mathrm{MoS}_{2}$ Electron mobility & $25 \mathrm{~cm}^{2} / \mathrm{Vs}$ \\
$\mathrm{MoS}_{2}$ Hole mobility & $25 \mathrm{~cm}^{2} / \mathrm{Vs}$ \\
$\mathrm{MoS}_{2}$ Saturation velocity & $0.28 \times 10^{7}$ \\
$\mathrm{Channel}_{\text {Doping }}$ & $6.5 \times 10^{7} \mathrm{~cm}^{-2}$ \\
source/drain region Doping & $6.5 \times 10^{12} \mathrm{~cm}^{-2}$ \\
$\mathrm{Resistivity} \mathrm{of} \mathrm{ferroelectric} \mathrm{layer}$ & $0.05 \Omega \mathrm{m}$ \\
\hline
\end{tabular}

\section{ANALYTICAL MODEL DEVELOPMENT}

An analytical model for the proposed negative capacitance $\mathrm{MoS}_{2}$ FET based biosensor is presented by considering the effect of drain coupling [32]. Two main regions have been considered for development of the model. Region-I is of 20 $\mathrm{nm}$ long and is filled with oxide layers and Region II is also $20 \mathrm{~nm}$ cavity which has been utilized for sensing biomolecules. A simple parallel plate capacitor model can be used to predict how the capacitances change with device dimensions since they rely on capacitive coupled gates. For simplification of the model the effect of fringing fields is neglected. The capacitances of Region I and Region II which are filled with oxide and biomolecules (or air) respectively are given as

$C_{o x}=\epsilon_{o x} / t_{o x}$

$C_{\text {bio }}=\epsilon_{\text {bio }} / t_{\text {bio }}$

$C_{\text {air }}=\epsilon_{\text {air }} / t_{\text {air }}$

where, $\epsilon_{o x}, \epsilon_{\text {bio }}, \epsilon_{\text {air }}$ are the dielectric constants of oxide, biomolecules and air respectively. Similarly, $t_{\mathrm{ox}}$ and $\mathrm{t}_{\mathrm{bio}}$ are the thickness of oxide layer and the cavity is filled with biomolecules/air respectively. For simplicity of the model we have considered $t_{o x}=t_{b i o}$. Hence the total effective capacitance of Region I and Region II is given by $C_{e f f}=$ $C_{o x}+C_{\text {bio }}$.

In order to develop the analytical model for $\mathrm{NC} \mathrm{MoS}_{2}$ FET biosensor the drain current $\left(\mathrm{I}_{\mathrm{ds}}-\mathrm{V}_{\mathrm{mos}}\right)$ and front-gate terminal charge $\left(\mathrm{Q}_{\mathrm{g}, \mathrm{mos}}-\mathrm{V}_{\mathrm{mos}}\right)$ models are coupled with $1 \mathrm{D}$ steady state Landau-Khalatnikov (L-K) equation as considered [33].

From the device structure the total gate voltage can be written as [35] 


$$
\begin{aligned}
V_{g s}=V_{m o s}+V_{N C} & =V_{m o s}+2 \alpha t_{N C} Q_{g, m o s}+4 \beta t_{N C} Q_{g, m o s}^{3} \\
& +6 \gamma t_{N C} Q_{g, m o s}^{5}
\end{aligned}
$$

where $\mathrm{V}_{\mathrm{mos}}$ and $\mathrm{V}_{\mathrm{NC}}$ are the internal metal-gate voltage and voltage drop across the ferroelectric material respectively. $\alpha$, $\beta$ and $\gamma$ are the ferroelectric material parameters and the values considered as provided in [36]. The $t_{\mathrm{NC}}$ is the thickness of ferroelectric material.

The front-gate terminal charge $\mathrm{Q}_{\mathrm{g}, \mathrm{mos}}$ for the $\mathrm{MoS}_{2}$ FET can be modelled by integrating the local channel charge along the channel [35]

$Q_{g, m o s}=\frac{1}{L_{g}} \int_{0}^{L_{g}} C_{e f f}\left[V_{m o s}-V_{F B}-\psi_{s}(x)\right] d x$

In order to consider the effect of drain coupling in the $\mathrm{Q}_{\mathrm{g}, \mathrm{mos}}-\mathrm{V}_{\text {mos }}$ model, the surface potential distribution $\psi_{s}(x)$ of the short channel $\mathrm{MoS}_{2}$ FET have been used. Now by applying Gauss's law to the $\mathrm{MoS}_{2}$ channel region [37]

$$
\begin{aligned}
& q N_{c h} \Delta x=t_{c h}\left[\varepsilon_{s} \overrightarrow{E_{s, x}}(x+\Delta x)-\varepsilon_{s} \overrightarrow{E_{s, x}}(x)\right]+ \\
& \Delta x\left[\varepsilon_{b o x} \overrightarrow{E_{b o x}}-\varepsilon_{s} \overrightarrow{E_{s, y}}\right]
\end{aligned}
$$

where $\varepsilon_{s}, \varepsilon_{\text {box }}$ are the permittivity of channel and buried oxide, respectively. $\mathrm{E}_{\mathrm{s}, \mathrm{x}}$ and $\mathrm{E}_{\mathrm{s}, \mathrm{y}}$ represent the vertical and lateral components of channel electric fields. Similarly, $\mathrm{N}_{\mathrm{ch}}$ and $t_{\text {ch }}$ are the channel doping concentration and thickness of channel, respectively. $E_{b o x}$ is the vertical field on the top of the buried oxide and can be represented as

$E_{\text {box }}=\left[V_{b s}-V_{f b b}-\psi_{s}(x)\right] / t_{b o x}$.

When there are no biomolecules inside the cavity the vertical electric field is given as

$$
E_{s, x}=\frac{\varepsilon_{o x}+\varepsilon_{a i r}}{\varepsilon_{s}}\left[\psi_{s}(x)-V_{g s}+V_{f b}\right] / t_{o x} .
$$

Similarly, with biomolecules inside the cavity the vertical electric field is given as

$$
E_{s, y}=\frac{\varepsilon_{o x}+\varepsilon_{b i o}}{\varepsilon_{s}}\left[\psi_{s}(x)-V_{g s}+V_{f b}\right] / t_{o x} \text {. }
$$

The lateral component of channel electric field is given as $d \psi_{s}(x) / d x$.

After substituting these relations into (5), we got the differential equation for the surface potential as follows [35] $l_{i}^{2} \frac{d^{2} \psi_{s}(x)}{d x^{2}}-\psi_{s}(x)+\psi_{s, \text { long }, i}=0$

with $\mathrm{i}=$ air or biomolecules.

Where $l_{i}$ is the characteristic length and is given as

$$
\begin{aligned}
& l_{\text {air }}=\left[\frac{\varepsilon_{s} t_{c h} t_{\text {box }} t_{o x}}{\left(\varepsilon_{o x}+\varepsilon_{\text {air }}\right) t_{\text {box }}+\varepsilon_{\text {box }} t_{o x}}\right]^{1 / 2} \\
& l_{\text {bio }}=\left[\frac{\varepsilon_{s} t_{c h} t_{\text {box }} t_{o x}}{\left(\varepsilon_{o x}+\varepsilon_{\text {bio }}\right) t_{\text {box }}+\varepsilon_{\text {box }} t_{o x}}\right]^{1 / 2}
\end{aligned}
$$$$
\psi_{s, l o n g, i} \text { is the long-channel surface potential and is given }
$$$$
\text { as }
$$

$\psi_{\text {s,long,air }}=$

$\frac{-q N_{c h} t_{o x} t_{b o x}+\left(\varepsilon_{o x}+\varepsilon_{a i r}\right) t_{b o x}\left(V_{\text {mos }}-V_{f b}\right)+\varepsilon_{b o x} t_{o x}\left(V_{b s}-V_{f b b}\right)}{\left(\varepsilon_{o x}+\varepsilon_{a i r}\right) t_{b o x}+\varepsilon_{\text {box }} t_{o x}}(10 . \mathrm{a})$

$\psi_{\text {s,long,bio }}=$

$\frac{-q N_{c h} t_{o x} t_{b o x}+\left(\varepsilon_{o x}+\varepsilon_{b i o}\right) t_{b o x}\left(V_{\text {mos }}-V_{f b}\right)+\varepsilon_{b o x} t_{o x}\left(V_{b s}-V_{f b b}\right)}{(10}$

$\left(\varepsilon_{o x}+\varepsilon_{\text {bio }}\right) t_{\text {box }}+\varepsilon_{\text {box }} t_{\text {ox }}$
Now considering the boundary condition $\psi_{s}(0)=V_{b i}$ and $\psi_{s}\left(L_{g}\right)=V_{b i}+V_{d s}$ the surface potential distribution can be solved as follows

$$
\begin{array}{r}
\psi_{s, i}(x)=\psi_{s, \text { long }, i}+\left(V_{b i}-\psi_{s, \text { long }, i}\right) \frac{\sinh \left[\left(L_{g}-x\right) / l_{i}\right]}{\sinh \left(L_{g} / l_{i}\right)} \\
+\left(V_{b i}+V_{d s}-\psi_{s, \text { long }, i}\right) \frac{\sinh \left(y / l_{i}\right)}{\sinh \left(L_{g} / l_{i}\right)}
\end{array}
$$

Now substituting (11) in (3) the front-gate terminal charge $\mathrm{Q}_{\mathrm{g} \text {, mos }}$ can be solved as follows

$$
\begin{aligned}
Q_{g, \text { mos,air }}=\left(C_{o x}\right. & \left.+C_{\text {air }}\right)\left[V_{\text {mos }}-V_{f b}-\psi_{s, \text { long,air }}\right] \\
& -\left(C_{o x}\right. \\
& \left.+C_{\text {air }}\right)\left[\frac { l _ { \text { air } } } { L _ { g } } \left(2 V_{b i}-2 \psi_{s, \text { long,air }}\right.\right. \\
& \left.\left.+V_{d s}\right) \frac{\cosh \left(L_{g} / l_{\text {air }}\right)-1}{\sinh \left(L_{g} / l_{\text {air }}\right)}\right]
\end{aligned}
$$

$$
\begin{aligned}
Q_{g, \text { mos }, \text { bio }}=\left(C_{o x}\right. & \left.+C_{\text {air }}\right)\left[V_{\text {mos }}-V_{f b}-\psi_{s, \text { long }, \text { bio }}\right] \\
& -\left(C_{o x}\right. \\
& \left.+C_{\text {bio }}\right)\left[\frac { l _ { \text { bio } } } { L _ { g } } \left(2 V_{b i}-2 \psi_{s, \text { long }, \text { bio }}\right.\right. \\
& \left.\left.+V_{d s}\right) \frac{\cosh \left(L_{g} / l_{b i o}\right)-1}{\sinh \left(L_{g} / l_{b i o}\right)}\right]
\end{aligned}
$$

From the surface potential the subthreshold drain current can be modelled as follows.

$I_{d s, i}=\frac{\mu_{n} Z K T\left(n_{i}^{2} / N_{c h}\right)\left[1-\exp \left(-q V_{d s} / K T\right)\right]}{\int_{0}^{L g}\left[\exp \left(-q \psi_{s, i}(x) / K T\right)\right] d x}$

The capacitance of $\mathrm{MoS}_{2}$ FET and NC-FET are connected in series with each other. Hence, there is a voltage drop across both the capacitances. Therefore, the threshold voltage of both can be added. Now the $\mathrm{V}_{\mathrm{T}}$ of the $\mathrm{NC} \mathrm{MoS}_{2}$ FET is linked to the threshold voltage of the underlying $\mathrm{MoS}_{2}$ FET by

$$
V_{T, i}=V_{T, M o S_{2}}+V_{N C, i} \cong V_{T, M o S_{2}}+V_{m o s}+2 \alpha t_{N C} Q_{g, m o s, i}
$$

with $\mathrm{i}=$ air or biomolecules

Now the sensitivity parameters can be written as

$$
\begin{aligned}
& \Delta V_{T}=V_{T, \text { bio }}-V_{T, \text { air }} \\
& \Delta \psi_{S}=\psi_{s, \text { bio }}-\psi_{S, \text { air }} \\
& \Delta I_{D S}=I_{D S, \text { bio }}-I_{D S, \text { air }}
\end{aligned}
$$

Where, $\Delta V_{T}, \Delta \psi_{s}$ and $\Delta I_{D S}$ are the change in threshold voltage, surface potential and drain current respectively.

Now, the SS of the device can be modelled as follows 
$S S_{N C, M O S_{2}}=S S_{\text {MoS }_{2}} \frac{1}{A_{V}}$

where, $A_{V}=\frac{\left|C_{N C}\right|}{\left|C_{N C}\right|+\left|C_{m o s, i}\right|}$

and $\mathrm{i}=$ air or biomolecules. The $\mathrm{Q}_{\mathrm{g}, \mathrm{mos}, \mathrm{i}}$ is varied with respect to different biomolecules, resulting a change of $C_{m o s, i}$ and SS.

\section{RESUlTS AND DISCUSSION}

In this section the results of analytical model is presented and calibrated with TCAD device simulation results of negative capacitance $\mathrm{MoS}_{2}$ FET-based biosensor. The effect of different structural parameters and design consideration on sensitivity of the biosensor is also discussed.

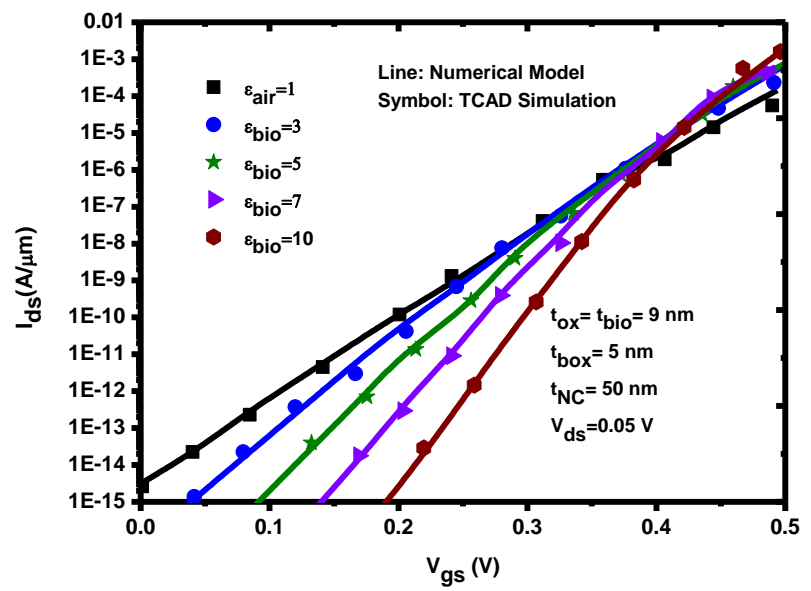

Fig. 2. Subthreshold $\mathrm{I}_{\mathrm{ds}}-\mathrm{V}_{\mathrm{gs}}$ characteristics of the NC-MoS FET for different biomolecules

When biomolecules are not present inside the cavity (air is filled with $\epsilon_{\text {air }}=1$ ), the effective gate capacitance decreases. On the other hand, when biomolecules are put inside the cavity, the dielectric constant of the cavity is modulated depending on the type of molecules $\left(\epsilon_{b i o}>1\right)$. The variations of gate capacitance bring about an effective capacitance coupling between gate and channel and hence there is a significant increase in threshold voltage as compared to air filled cavity. Fig. 2 shows TCAD device simulation and analytical model results of input transfer characteristics of $\mathrm{NC} \mathrm{MoS}_{2}$ FET biosensor for different biomolecules. From Fig. 2 it is observed that the threshold voltage increases with increase of dielectric constant of biomolecules. The sensitivity of the biosensor is defined as $\Delta V_{T}=V_{T, b i o}-V_{T, a i r}$. The change in threshold voltage has an inverse relationship with the permittivity of the biomolecules. Hence, larger the value of $\Delta V_{T}$ the better is the sensitivity. Illustrated in Fig. 1 (a), it is observed that with the introduction of biomolecules inside the cavity the threshold voltage shifts towards higher gate voltage. It is observed that the slope of the $\mathrm{Q}_{\mathrm{g}, \mathrm{mos}}-\mathrm{V}_{\mathrm{mos}}$ curve (i.e. $\mathrm{C}_{\mathrm{mos}}$ ) in the subthreshold region increases with increasing dielectric constant of the biomolecules. The dielectric constant dependence of our $\mathrm{Q}_{\mathrm{g}, \mathrm{mos}}-\mathrm{V}_{\mathrm{mos}}$ model can be observed in Eq.12 (b). The slope is different for different biomolecules and all the curves meet at a point at a gate voltage of $0.4 \mathrm{~V}$, as shown in Fig. 2.
Channel doping concentration is an important device parameter for responsivity improvement of biosensor with low power consumption. In order to estimate the optimum value of channel doping concentration, it is varied in the simulation process. Fig. 3 shows that plot of threshold voltage shift $\left(\Delta V_{T}\right)$ versus channel doping concentration. The threshold voltage of the device can be modulated by both channel doping concentration and biomolecule absorption by changing gate capacitance. When the channel doping concentration is low, the channel depletes more and hence the threshold voltage of the device increases even before biomolecules absorption. This concludes that for low doped channel the device has less responsivity even for higher dielectric constant biomolecules due to increment of depletion width. Hence, the biomolecules are splitted into smaller molecules resulting reduction of sensitivity. When the channel doping concentration increases, there is a significant increase in sensitivity of the device. From Fig. 3 it is observed that with small increase in channel doping, the biomolecules are absorbed inside the cavity and a significant threshold voltage variation is observed.

With further increase in channel doping concentration, the sensitivity of the device decreases for low dielectric constant biomolecules, but at the same time increases the segregation potency of the device for variety of biomolecules. In case of highly doped $\mathrm{MoS}_{2}$ FET and before the absorption of biomolecules the depletion width below the gate is reduced which makes it more difficult to the channel to fully deplete. Hence, the off-state current and static power consumption of the device increases. In case of highly doped $\mathrm{MoS}_{2}$ FET and after the absorption of biomolecules the channel doping concentration is more dominant on the threshold voltage than the absorbed biomolecules. In this situation, it is very difficult to consider threshold voltage shift as sensitivity parameter. So, in order to design a low power high sensitive biosensor, optimum value of channel doping concentration plays an important role. From the graph the optimum value of channel doping is found as $2.8 \times 10^{18}$ $\mathrm{cm}^{-3}$ for our proposed low power biosensor.

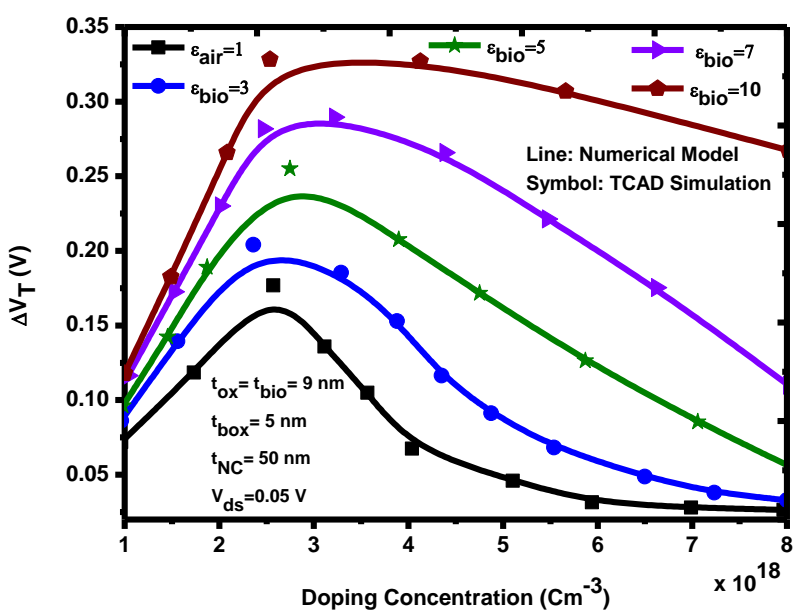

Fig. 3. Threshold voltage shift versus channel doping concentration for different biomolecules

Fig. 4 shows the $\mathrm{Q}_{\text {gmos }}-\mathrm{V}_{\text {mos }}$ characteristics of the device for different biomolecules obtained through both analytical model and device simulation. From the graph it is observed 
that the slope of the $\mathrm{Q}_{\mathrm{g} \text {,mos }}-\mathrm{V}_{\text {mos }}$ curve (i.e. $\mathrm{C}_{\mathrm{mos}}$ ) increases with increase in dielectric constant of the biomolecules. Hence, subthreshold slope of the device also increases with increase in dielectric constant. This increment is due to the increase of drain coupling $\left(\mathrm{C}_{\mathrm{DIBL}}\right)$ through high-dielectric biomolecules. It is concluded that the sensitivity of the devices increases with increase in dielectric constant of the biomolecule. But at the same time nonlinearity is observed in subthreshold I-V and average SS curve due to the negative capacitance which is a strong bias dependent.

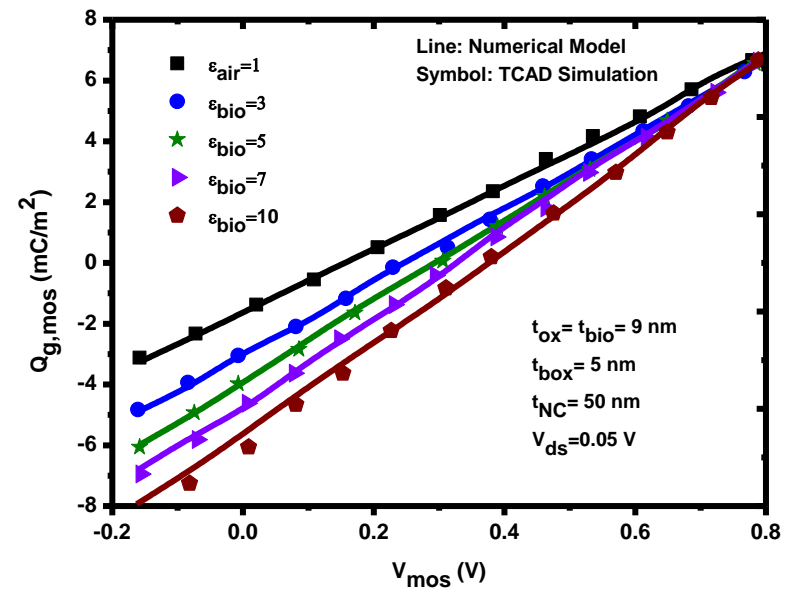

Fig. 4. $\mathrm{Q}_{\mathrm{g}, \mathrm{mos}}-\mathrm{V}_{\mathrm{mos}}$ characteristic of $\mathrm{NC}-\mathrm{MoS}_{2}$ FET for different biomolecules

Fig. 5 shows the plot between threshold voltage and gate length of the device. It is observed from the graph that the threshold voltage increases with decrease in gate length which is quite different from conventional MOSFET. When the gate length decreases the $\mathrm{V}_{\mathrm{NC}}\left(\sim 2 \alpha \mathrm{t}_{\mathrm{NC}} \mathrm{Q}_{\mathrm{g}, \mathrm{mos}}\right)$ becomes more positive as $\mathrm{Q}_{\mathrm{g}, \mathrm{mos}}$ decreases. Again $V_{T, i}=V_{T, \text { MoS }_{2}}+$ $V_{N C, i}$, since $\mathrm{V}_{\mathrm{NC}}$ increases with decrease of gate length the threshold voltage of the device also increases. The graph also shows that with increase in dielectric constant of the biomolecule the threshold voltage also increases ( $\mathrm{V}_{\mathrm{NC}}$ becomes more positive since $\mathrm{Q}_{\mathrm{g}, \mathrm{mos}}$ decreases).

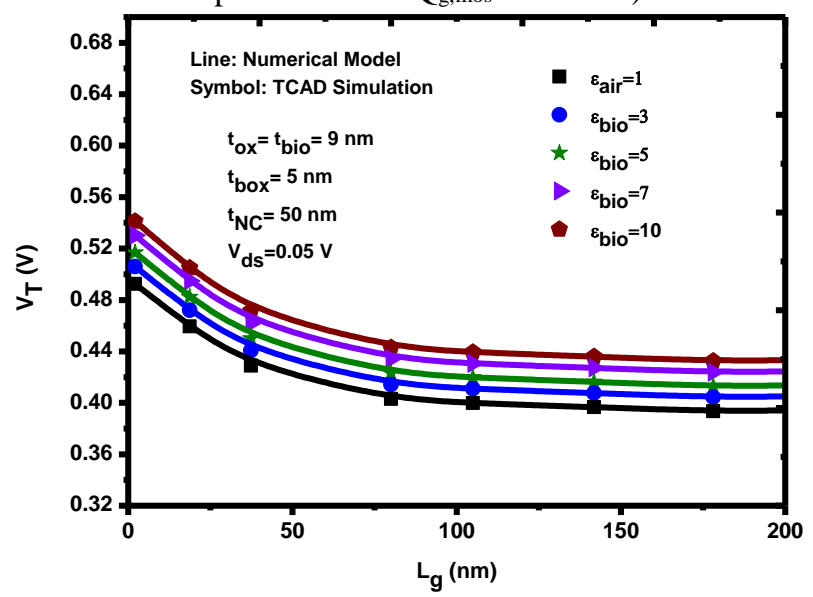

Fig. 5. Threshold voltage shift versus gate length for different biomolecules

From Fig. 2 - Fig. 5, it is observed that the analytical model and TCAD device simulation results are reasonably matched with each other which validates our proposed model.

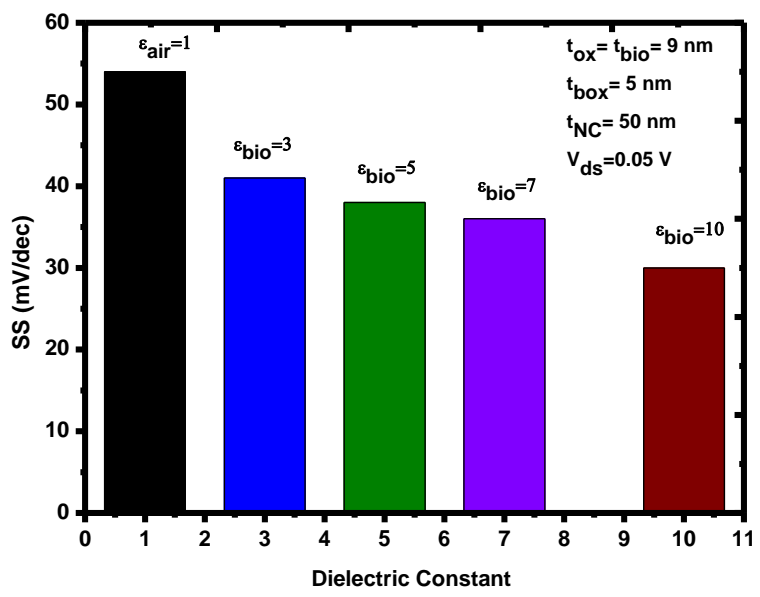

Fig. 6. SS of NC $\mathrm{MoS}_{2}$ FET biosensor for different biomolecules

In order to design any biosensor, one of the most important parameters is the sensing speed of the device. The sensing speed of the biosensor indicates that how fast the biomolecules in the cavity can be identified. In order to achieve this SS must be as low as possible. When the SS of the device is low then a lesser input voltage change is required for changing one decade of output current. So when the surface potential in the $\mathrm{MoS}_{2}$ channel changes due to change of biomolecules in the cavity, it shows a sudden change in output characteristics of the device and results enhanced sensing speed. On the other hand, if the SS of the device is higher, then a larger input voltage change is required for changing the output current and hence sensing speed of the biosensor is also lower. In our proposed NC $\mathrm{MoS}_{2}$ FET based biosensor, the SS of the device is lower due to the presence of ferroelectric material below the gate and hence the sensing speed also increases. In order to show the sensing speed of the proposed biosensor the extracted SS of different biomolecules inside the cavity are presented in Fig. 6. It shows that when the dielectric constant of the biomolecules increases then the SS of the device decreases. Fig. 7 shows sensitivity of the biosensor in terms of SS. From this it is concluded that the proposed biosensor is more sensitive to higher dielectric constant biomolecules. From the figure, it is also observed that the SS of our proposed biosensor is relatively lower (below $60 \mathrm{mV} / \mathrm{dec}$ ) as compared to conventional biosensor. So, it is concluded that the proposed device can be applicable for low power biosensor.

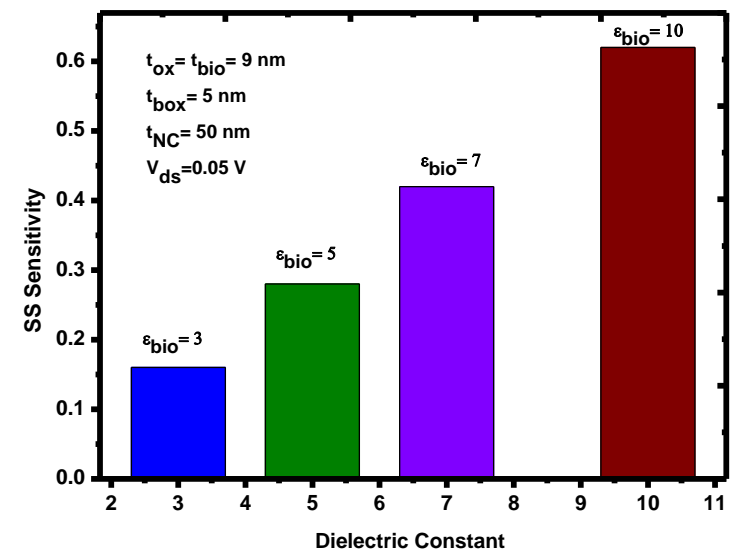

Fig. 7. SS sensitivity of $\mathrm{NC} \mathrm{MoS}_{2}$ FET biosensor for different biomolecules 
Fig. 8 shows $\mathrm{I}_{\mathrm{on}} / \mathrm{I}_{\text {off }}$ ratio of the proposed biosensor for different biomolecules. From this figure it is observed that the ratio increases with increase in dielectric constant of the biomolecules. Since the 'off' state current is constant and the 'on' current increases with increase in dielectric constant, the ratio increases. So it is concluded that the proposed biosensor has higher sensitivity in terms of $\mathrm{I}_{\text {on }} / \mathrm{I}_{\text {off }}$ ratio.

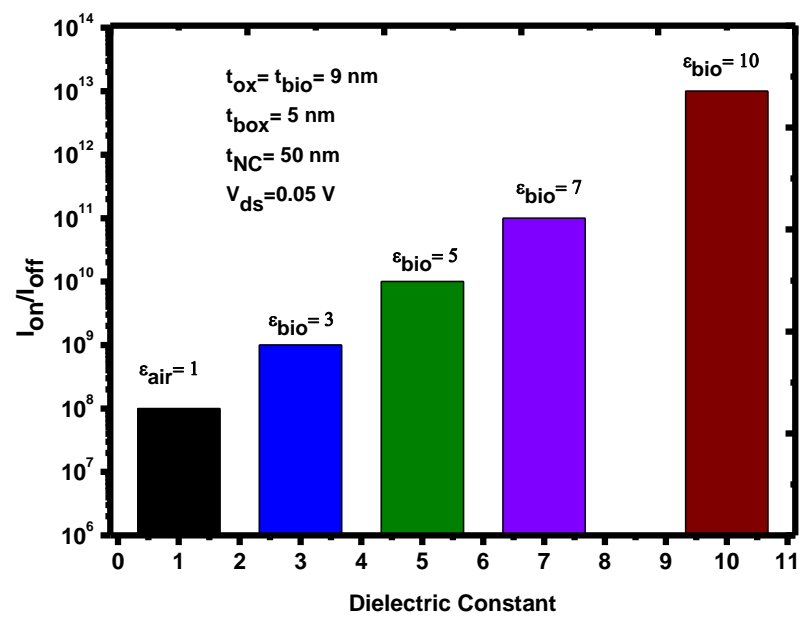

Fig. 8. $\mathrm{I}_{\mathrm{on}} / \mathrm{I}_{\mathrm{off}}$ ratio of $\mathrm{NC} \mathrm{MoS}{ }_{2}$ FET biosensor for different biomolecules

In order to estimate the sensitivity of the biosensor in terms of drain current, the sensitivity is expressed as

$$
S=\frac{\Delta I_{d s}}{I_{d s}} \times 100=\frac{\left|I_{d s, \text { bio }}-I_{d s, \text { air }}\right|}{\min \left(I_{d s, \text { bio }}, I_{d s, \text { air }}\right)} \times 100
$$

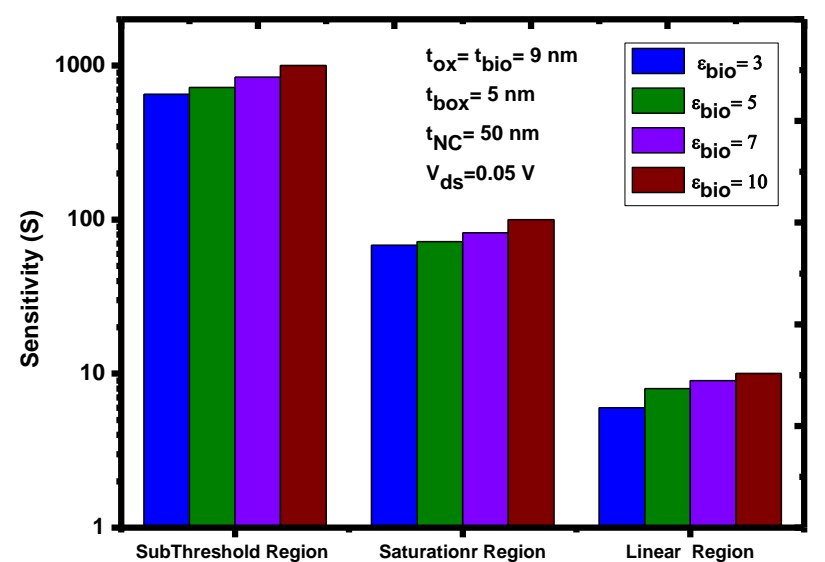

Fig. 9. Drain current sensitivity of $\mathrm{NC} \mathrm{MoS}_{2}$ FET biosensor for different regions of operation

Fig. 9 shows the sensitivity curve of the proposed NC $\mathrm{MoS}_{2}$ FET biosensor in all regions of operation. From the figure it is observed that the device shows highest sensitivity in the subthreshold region and the sensitivity decreases gradually when we move to linear region. Hence, it is concluded that, in order to obtain maximum sensitivity, the proposed biosensor should be operated in subthreshold region. The presented analytical model is developed in subthreshold region only.

Table II presents the comparison of different sensitivity parameters between $\mathrm{NC} \mathrm{MoS}$ FET biosensor and $\mathrm{MoS}_{2}$ FET biosensor. From the table it is observed that by inserting a ferroelectric material below the gate, the sensitivity of the device increases due to NC effect. From the above discussion, it is concluded that, due to the effect of drain coupling $\left(\mathrm{C}_{\mathrm{DC}}\right)$ on the negative-capacitance effect, the $\mathrm{NC}$ $\mathrm{MoS}_{2}$ FET shows enhanced short-channel effects such as improved average SS and $\mathrm{I}_{\mathrm{on}} / \mathrm{I}_{\text {off }}$ ratio with decreasing gate length.

Table II. DifFERENT SENSITIVITy PARAMETERS COMPARISON BETWEen $\mathrm{NC} \mathrm{MoS}_{2}$ FET Biosensor AND $\mathrm{MOS}_{2}$ FET BIOSENSOR IN SUBTHRESHOLD REGION

\begin{tabular}{ccccc}
\hline Parameters & $\begin{array}{c}\text { Drain current } \\
\text { Sensitivity } \\
(\mathrm{S})\end{array}$ & $\Delta \mathrm{V}_{\mathrm{T}}$ & $\begin{array}{c}\mathrm{SS} \\
(\mathrm{mVV} / \mathrm{dec})\end{array}$ & $\mathrm{I}_{\text {on }} / \mathrm{I}_{\text {off }}$ \\
\hline NC-MoS $\mathbf{2}$ FET & & & & \\
$\varepsilon_{\text {bio }}=3$ & 650 & 0.18 & 41 & $10^{9}$ \\
$\varepsilon_{\text {bio }}=5$ & 720 & 0.23 & 38 & $10^{10}$ \\
$\varepsilon_{\text {bio }}=7$ & 840 & 0.28 & 36 & $10^{11}$ \\
$\boldsymbol{\varepsilon}_{\text {bio }}=\mathbf{1 0}$ & $\mathbf{1 0 0 0}$ & $\mathbf{0 . 3 4}$ & $\mathbf{3 0}$ & $\mathbf{1 0}^{\mathbf{1 3}}$ \\
$\mathbf{M o S _ { 2 }} \mathbf{F E T}$ & & & & \\
$\varepsilon_{\text {bio }}=3$ & 470 & 0.11 & 59 & $10^{4}$ \\
$\varepsilon_{\text {bio }}=5$ & 560 & 0.13 & 55 & $10^{5}$ \\
$\varepsilon_{\text {bio }}=7$ & 630 & 0.14 & 50 & $10^{6}$ \\
$\boldsymbol{\varepsilon}_{\text {bio }}=\mathbf{1 0}$ & $\mathbf{7 8 0}$ & $\mathbf{0 . 1 6}$ & $\mathbf{4 2}$ & $\mathbf{1 0}^{\mathbf{8}}$ \\
\hline
\end{tabular}

\section{CONCLUSION}

In this work a dielectric modulated negative capacitance (NC) $\mathrm{MoS}_{2}$ FET based biosensor is proposed for electrical detection of different biomolecules. The performance of the biosensor is investigated in terms of drain current, threshold voltage shift, $\mathrm{I}_{\mathrm{on}} / \mathrm{I}_{\text {off }}$ ratio, $\mathrm{SS}$ and sensitivity. From the simulation results, it is observed that the proposed biosensor obtained maximum sensitivity, when the device is operated in subthreshold region. An analytical model is developed for the proposed biosensor in subthreshold region and validated with TCAD simulation results. The developed analytical model provides a clear understanding of physics of $\mathrm{NC}-\mathrm{MoS}_{2}$ FET based biosensor for detection of targeted biomolecules. It is also concluded that $\mathrm{NC}-\mathrm{MoS}_{2}$ FET biosensor shows enhanced sensitivity as compared to conventional $\mathrm{MoS}_{2}$ FET, due to the NC effect of ferroelectric material which shows improved short channel effects such as enhanced average $S S$ and $\mathrm{I}_{\text {on }} / \mathrm{I}_{\text {off }}$ ratio with scale down of gate length. Additionally, the sensing of high dielectric constant biomolecules inside the cavity further enhances the sensitivity and subthreshold characteristics of the short channel device and hence consumes less power. Owing to the high sensitivity and low power of the proposed biosensor, the device can be applicable in medical diagnostics, forensic industries, national security, and environment monitoring.

\section{ACKNOWLEDGMENTS}

The authors acknowledge VIT-AP University for facilitating to carry out this research. Acknowledgment also goes to National Institute of Technology Silchar, GLA University Mathura, Kuwait College of Science and Technology and New Jersey Institute of Technology (NJIT) Newark, USA for the collaboration to carry out the research work.

\section{REFERENCES}


[1] D. Moon, J. Han and M. Meyyappan, "Comparative Study of Field Effect Transistor Based Biosensors," IEEE Transactions on Nanotechnology, vol. 15, no. 6, pp. 956-961, Nov. 2016.

[2] K. Xu, "Integrated Silicon Directly Modulated Light Source Using p-Well in Standard CMOS Technology," IEEE Sensors Journal, vol. 16, no. 16, pp. 6184-6191, Aug.15, 2016,

[3] By Yi Cui, Qingqiao Wei, Hongkun Park, Charles M. Lieber, "Nanowire Nanosensors for Highly Sensitive and Selective Detection of Biological and Chemical Species," Science, vol. 293, no. 5533, pp. 1289-1292, Aug 2001.

[4] R. Narang, M. Saxena, M. Gupta, "Comparative analysis of dielectric-modulated FET and TFET-based biosensor," IEEE Transactions on Nanotechnology, vol.14, no.3, pp. 427-435, 2015.

[5] X. Gong, R. Zhao and X. Yu, "A 3-D-Silicon Nanowire FET Biosensor Based on a Novel Hybrid Process," Journal of Microelectromechanical Systems, vol. 27, no. 2, pp. 164-170, April 2018

[6] R. Mas-Balleste, C. Gomez-Navarro, J. Gomez-Herrero, and F. Zamora, "2D materials: To graphene and beyond," Nanoscale, vol.3, pp.20-30, 2011

[7] K Sun et al., "Effect of subthreshold slope on the sensitivity of nanoribbon sensors," Nanotechnology, vol. 27, pp.288501, 2016.

[8] X. Zou, J. Xu, H. Huang, Z. Zhu, H. Wang, B. Li, L. Liao, and G. Fang, "A comparative study on top-gated and bottom-gated multilayer $\mathrm{MoS}_{2}$ transistors with gate stacked dielectric of $\mathrm{Al}_{2} \mathrm{O}_{3} / \mathrm{HfO}_{2}$," Nanotechnology, vol. 29, no.24, pp. 245201, 2018.

[9] W. Sik Hwang et al., "Transistors with chemically synthesized layered semiconductor WS2 exhibiting 105 room temperature modulation and ambipolar behaviour," Appl. Phys. Lett., vol. 101, pp. 013107, 2012

[10] H. Fang, S. Chuang, T. C. Chang, K. Takei, T. Takahashi, and A. Javey, "High-performance single layered $\mathrm{WSe}_{2}$ p-FETs with chemically doped contacts," Nano Lett., vol.12, pp.37883792, 2012.

[11] Yariv I, Lipovsky A, Gedanken A, Lubart R, Fixler D. "Enhanced pharmacological activity of Vitamin B12 and Penicillin as nanoparticles," Int J. Nanomedicine. vol.10, no.1, pp.3593-3601, 2015.

[12] Adam Bolotsky et al., "Two-Dimensional Materials in Biosensing and Healthcare: From in Vitro Diagnostics to Optogenetics and Beyond," ACS Nano, vol.13, pp. 9781-9810, 2019.

[13] Kuanchen Xiong1 et al. "CMOS-compatible Batch Processing of Monolayer $\mathrm{MoS}_{2}$ MOSFETs," Journal of Physics D: Applied Physics, vol.51, No.15, pp. 51, 2018.

[14] Y. Yoon, K. Ganapathi, and S. Salahuddin, "How good can monolayer $\mathrm{MoS}_{2}$ transistors be?," Nano Lett., vol. 1, pp. 3768 3773, 2011.

[15] J. Jo, W. Y. Choi, J. D. Park, J. W. Shim, H. Y. Yu, and C. Shin, "Negative capacitance in organic/ferroelectric capacitor to implement steep switching MOS devices," Nano Lett., vol. 15, pp.4553-4556, 2015.

[16] J. H. Park et al., "Sub-kT/q subthreshold-slope using negative capacitance in low-temperature polycrystalline silicon thinfilm transistor," Sci. Rep., vol.6, pp.24734, 2016.

[17] J. Jo and C. Shin, "Negative capacitance field effect transistor with hysteresis-free sub-60 mV/decade switching," IEEE Electron Device Lett. Vol.37, pp.245-248, 2016.

[18] X. Wang et al., "Two-dimensional negative capacitance transistor with polyvinylidene fluoride-based ferroelectric polymer gating," npj 2D Mater. Appl. vol.38, 2017.

[19] X. Liu et al., "MoS2 negative capacitance field effect transistors with subthreshold swing below the physics limit," Adv. Mater,vol.30, pp.1800932, 2018.

[20] J. Xu, S. Y. Jiang, M. Zhang, H. Zhu, L. Chen, Q. Q. Sun, and D. W. Zhang, "Ferroelectric HfZrOx-based $\mathrm{MoS}_{2}$ negative capacitance transistor with ITO capping layers for steep-slope device application,” Appl. Phys. Lett., vol. 112, pp. 103104, 2018.

[21] A. Nourbakhsh, A. Zubair, S. Joglekar, M. Dresselhaus, and T. Palacios, "Subthreshold swing improvement in $\mathrm{MoS}_{2}$ transistors by the negative-capacitance effect in a ferroelectric Al-doped-HfO2/HfO2 gate dielectric stack," Nanoscale, vol.9, pp.6122-6127, 2017.

[22] F. A. McGuire, Y. C. Lin, K. Price, G. B. Rayner, S. Khandelwal, S. Salahuddin, and A. D. Franklin, "Sustained sub-60 $\mathrm{mV} /$ decade switching via the negative capacitance effect in $\mathrm{MoS}_{2}$ transistors," Nano Lett., vol. 17, pp. 4801-4806, 2017.

[23] Late, D. J., Huang, Y.-K., Liu, B., Acharya, J., Shirodkar, S. N., Luo, J., Yan, A., Charles, D., Waghmare, U. V., Dravid, V. P.," Sensing Behavior of Atomically Thin-Layered $\mathrm{MoS}_{2}$ Transistors." ACS Nano, vol.7, pp. 4879-4891, 2013.

[24] Deblina Sarkar, Wei Liu, Xuejun Xie, Aaron C. Anselmo, Samir Mitragotri, and Kaustav Banerjee, "MoS 2 Field-Effect Transistor for NextGeneration Label-Free Biosensors," ACS nano, vol.. 8, no. 4, pp. 3992-4003, 2014.

[25] Kim, C. H., Jung, C., Park, H. G., \& Choi, Y. K., "Novel dielectric modulated field-effect transistor for label-free DNA detection," Biochip J, vol. 2, no.2, pp. 127-134, 2008.

[26] Narang, R., Reddy, K. S., Saxena, M., Gupta, R. S., \& Gupta, M., "A dielectric-modulated tunnel-FET-based biosensor for label-free detection: Analytical modelling study and sensitivity analysis." IEEE transactions on electron devices, vol.59, no.10, pp.2809-2817, 2012.

[27] Singh, D., Pandey, S., Nigam, K., Sharma, D., Yadav, D. S., \& Kondekar, P. "A charge-plasma-based dielectric-modulated junctionless TFET for biosensor label-free detection." IEEE Transactions on Electron Devices, vol. 64, no.1, pp. 271-278, 2016.

[28] W. Cao, J. Kang, W. Liu, and K. Banerjee, "A compact current-voltage model for 2D semiconductor based field-effect transistors considering interface traps, mobility degradation, and inefficient doping effect," IEEE Trans. Electron Devices, vol. 61, no. 12, pp. 4282-4290, Dec. 2014

[29] W.-X. You and P. Su, "A compact subthreshold model for short-channel monolayer transition metal dichalcogenide field-effect transistors," IEEE Trans. Electron Devices, vol. 63, no. 7, pp. 2971-2974, Jul. 2016.

[30] Felicia A. McGuire, Zhihui Cheng et al.," Sub- $60 \mathrm{mV} /$ decade switching in 2D negative capacitance field-effect transistors with integrated ferroelectric polymer," Appl. Phys. Lett. 109, 093101 (2016)

[31] ATLAS User's Manual, SILVACO International, 2019.

[32] Xu, K., "Silicon MOS Optoelectronic Micro- Nano Structure Based on Reverse- Biased PN Junction.” Phys. Status Solidi A, vol. 216, 2019

[33] L. D. Landau and I. M. Khalatnikov, "On the anomalous absorption of sound near a second order phase transition point," Dokl. Akad. Nauk SSSR, vol. 96, pp. 469-472, Jun. 1954.

[34] X. Wang et al., "Two-dimensional negative capacitance transistor with polyvinylidene fluoride-based ferroelectric polymer gating," 2D Mater. Appl., vol. 1, no. 38, pp. 1-7, Nov. 2017.

[35] Y. Taur, J. Wu, and J. Min, "A short-channel I-V model for 2D MOSFETs," IEEE Trans. Electron Devices, vol. 63, no. 6, pp. 2550-2555, Jun. 2016.

[36] K. M. Rabe, M. Dawber, C. Lichtensteiger, C. H. Ahn, and J. M. Triscone, "Modern physics of ferroelectrics: Essential background," Physics of Ferroelectrics (Springer, Berlin, Heidelberg), pp. 1-30, 2007.

[37] C. Hong et al., "A general and transformable model platform for emerging multi-gate MOSFETs," IEEE Electron Device Lett., vol. 38, no. 8, pp. 1015-1018, Aug. 2017. 